\title{
Reversible and irreversible magnetocaloric effect: the cases of rare-earth intermetallics $\mathrm{YbPt}_{2} \mathrm{Sn}$ and $\mathrm{Ce}_{0.5} \mathrm{La}_{0.5} \mathrm{~B}_{6}$
}

\author{
Thomas Gruner ${ }^{1}$, Daeho Kim², Manuel Brando ${ }^{3}$, Anatoliy V. Dukhnenko \\ Natalya Y. Shitsevalova ${ }^{4}$, Volodymyr B. Filipov ${ }^{4}$, and Dongjin Jang ${ }^{2 *}$ \\ ${ }^{1}$ Cavendish Laboratory, Department of Physics, University of Cambridge, Cambridge CB3 OHE, United Kingdom \\ ${ }^{2}$ Center for Thermometry and Fluid Flow Metrology, Division of Physical Metrology, \\ Korea Research Institute of Standards and Science (KRISS), 34113 Daejeon, Republic of Korea \\ ${ }^{3}$ Max Planck Institut für Chemische Physik fester Stoffe, 01187 Dresden, Germany \\ ${ }^{4}$ I. M. Frantsevich Institute for Problems of Materials Science of NAS, 3 Krzhyzhanovsky Street, Kiev 03680, Ukraine
}

\begin{abstract}
Magnetocaloric effect (MCE) has drawn much attention because its magnetic cooling property enables refrigeration without producing noxious gas or using rapidly depleting resources. However, applications for everyday life are yet distant. In addition, we need to understand more about the practical aspect of the MCE. Here, we introduce a phenomenological model to explain the quasiadiabatic MCE. Correction factors to the equilibrium thermodynamic feature implied by the entropy landscape are devised in analytic forms. To demonstrate the validity of the model, the MCE from two different materials is investigated. The recently discovered metallic paramagnet, $\mathrm{YbPt}_{2} \mathrm{Sn}$, shows a linear and reversible MCE which is typical of a paramagnetic system and suitable for cryogenics without ${ }^{3} \mathrm{He}$. On the other hand, a complex-phase material, $\mathrm{Ce}_{0.5} \mathrm{La}_{0.5} \mathrm{~B}_{6}$, exhibits a pronounced irreversible MCE especially across a magnetic phase boundary. A term that describes the field induced heating near a phase transition turns out to be essential in resolving the irreversible, non-equilibrium MCE.
\end{abstract}

\section{INTRONDUCTION}

Ever since it was first reported by Piccard and Weiss about hundred years ago, the magnetocaloric effect (MCE) has been defined as an adiabatic change of material's temperature in response to an external magnetic field $[1,2]$. Debye and Giauque independently proposed a concept of refrigeration inspired by the property that the adiabatic temperature of paramagnetic insulators decreases with external magnetic field [3-6]. In this way, the MCE is best known as the working principle of an adiabatic demagnetization refrigerator (ADR) [7-12]. Although an ideal magnetocaloric material (MCM) for cryogenic refrigeration is a robust metallic paramagnet with a large volumetric entropy capacity [12], the very low base temperatures of insulating paramagnets have been so advantageous when building an ADR that it overwhelms the shortcomings of an insulator. So far, the low thermal conductivity of an insulating MCM was supplemented by making a very tight contact between the MCM, the highly conducting metal parts and the protection canister $[8,9,11,13]$.

In accordance with surging demands for environmentally friendly devices, the research field searching for new MCMs has been greatly expanded from the early 2000's [14]. Until recently, many materials have been mentioned as candidates to build a demagnetization refrigerator at various temperature ranges [14-17].

For the fundamental research, the MCE is often scrutinized to determine a magnetic phase transition and phase diagram [18-22]. When we conduct an experiment, however, it is not feasible to create perfectly adiabatic conditions. Hence, researchers have mostly relied on heat capacity and magnetization measurements to estimate the MCE in the framework of equilibrium thermodynamics. In contrast, results from a direct field-sweep in a

* corresponding author, E-mail:dongjin.jang@kriss.re.kr quasi-adiabatic environment reveal richer traits. For example, for a certain complex-phase material in which many different magnetic order parameters are competing, non-equilibrium features arising from quasi-particle scatterings on the brink of a phase transition are pronounced [22]. Therefore, caution has to be paid in believing that equilibrium thermodynamic characteristics regarding the MCE can always be approximated even if a perfectly adiabatic field-sweep is possible.

In this paper, a practical model for the non-equilibrium MCE is introduced. The equation is developed by adding quasi-adiabatic, non-equilibrium and irreversible corrections to the equilibrium equation for the MCE. By using this model, two different MCMs are investigated. One is $\mathrm{YbPt}_{2} \mathrm{Sn}$, which is recently discovered metallic paramagnet suitable for building an ADR of $0.2 \mathrm{~K}$ base temperature [12]. The material shows a linear and reversible MCE, and additional non-equilibrium correction terms were not necessary. The other material is $\mathrm{Ce}_{1-x} \mathrm{La}_{x} \mathrm{~B}_{6}$, whose quantum mechanical state consists of at most five different multipolar phases depending on $x$, temperature $T$, and external magnetic field $B[22]$. The MCE observed in $\mathrm{Ce}_{0.5} \mathrm{La}_{0.5} \mathrm{~B}_{6}$ by a quasi-adiabatic field-sweep is analyzed in detail because its phase diagram is relatively simple. In this system, non-equilibrium effects are superposed with an equilibrium, reversible MCE observed from an entropy landscape.

\section{THEORY}

\section{A phenomenological model for the quasi-adiabatic MCE}

The magnetocaloric effect is usually defined as an adiabatic variation of material temperature with external magnetic field. In a perfectly adiabatic condition, we do not expect a change of an entropy $S$ over the $T$ - $B$ plane, and the following relation between infinitesimal variables 
should be satisfied in the framework of equilibrium thermodynamics,

$$
\left\{\begin{array}{l}
d S=\left(\frac{\partial S}{\partial T}\right)_{B} d T+\left(\frac{\partial S}{\partial B}\right)_{T} d B \\
d S=0 \text { (for an adiabatic process). }
\end{array}\right.
$$

By using Maxwell's relation between $S$ and $M$ (magnetization), $(\partial S / \partial B)_{T}=(\partial M / \partial T)_{B}$, and the general thermodynamic relation for heat capacity, $C_{B}=$ $(\partial Q / \partial T)_{B}=T(\partial S / \partial T)_{B}$, Eq. (1) can be reconstructed to give a rate of change of $T$ with $B$ in an adiabatic (or isentropic) condition,

$$
\left(\frac{\partial T}{\partial B}\right)_{S}=-\frac{T}{C_{p}(T, B)}\left(\frac{\partial M(T, B)}{\partial T}\right)_{B} .
$$

Since a material will be inspected under constant pressure, $C_{B}$ is expressed as $C_{p}(T, B)$. Eq. (2) is a textbook expression to describe the MCE [19]. As a representative example, let us solve the problem of an ideal paramagnet with total angular momentum $J$ [23]. In this case,

$$
C_{p}(T, B)=k_{\mathrm{B}} x^{2} \frac{\partial}{\partial x} B_{J}(x)
$$

and

$$
\left(\frac{\partial M(T, B)}{\partial T}\right)_{B}=-\frac{k_{\mathrm{B}}}{B} x^{2} \frac{\partial}{\partial x} B_{J}(x),
$$

where $k_{\mathrm{B}}$ is the Boltzmann constant, $x$ is equal to $g \mu_{B} B / k_{\mathrm{B}} T$, and $B_{J}(x)$ is the Brillouin function. Substitution of Eqs. (3) and (4) into Eq. (2) leads us to the differential equation which can be solved by the separation of variables,

$$
\begin{aligned}
\left(\frac{\partial T}{\partial B}\right)_{S} & =\frac{T}{B} \\
& \Rightarrow \int_{T_{\mathrm{i}}}^{T_{\mathrm{f}}} \frac{1}{T} d T=\int_{B_{\mathrm{i}}}^{B_{\mathrm{f}}} \frac{1}{B} d B \\
& \Rightarrow T_{\mathrm{f}}=\frac{T_{\mathrm{i}}}{B_{\mathrm{i}}} B_{\mathrm{f}}
\end{aligned}
$$

with subscripts $\mathrm{i}$ and $\mathrm{f}$ indicating initial and final values for the integration range. Now, we have arrived at the famous result that the demagnetization cooling curve of an ideal paramagnet is a straight line crashing to absolute zero over the $T$ - $B$ plane. However, the ideal solution violates the $3^{\text {rd }}$ law of thermodynamics which states that a system should have the same entropy as the temperature approaches to absolute zero even if an external variable such as $B$ is changed in the adiabatic state. In a practical paramagnet, ordering of spins occurs when small exchange interactions dominate thermal energy at very low temperatures. The similar ordering feature is often described by presuming a remnant magnetic field, $B_{\text {rem }}$, remaining inside the non-interacting spin system at $B_{\mathrm{f}}=0$. In this context, $T_{\mathrm{f}}$ is approximated as $T_{\mathrm{f}}=\left(T_{\mathrm{i}} / B_{\mathrm{i}}\right)\left(B_{\mathrm{f}}+B_{\text {rem }}\right)$.

As such, solving Eq. (2) might be the best way to predict the MCE if analytic expressions for $C_{p}(T, B)$ and $M(T, B)$ were known. However, there are not many exactly solvable quantum statistical models [24]. Also, celebrated mathematical techniques (see Eq. (5)) cannot be applied to all problems, e.g. it is not a general statement that the variables in the right-hand side (r.h.s.) of Eq. (2) are always separable. In addition, numerical evaluation of the r.h.s. of Eq. (2) is not always efficient. First of all, the procedure is time consuming because we need very fine measurements for both $C_{p}$ and $M$. Then, we need to estimate $\partial M / \partial T$ divided by $C_{p}$. It means high sensitivity of the result to a priori truncation error corresponding to derivatives and divisions even before integrating Eq. (2). Thus, a judicious approach might be needed in order to evaluate the r.h.s. of Eq. (2).

On the other hand, once $C_{p}(T, B)$ is measured in good resolution, the entropy, $S(T, B)=\int\left(C_{p} / T\right) d T$, can be calculated. An isentropic contour extracted from the $2 \mathrm{D}$ function, $S(T, B)$, is simply $T_{\mathrm{ad}}(B)$, the adiabatic temperature of a specimen as a function of $B$. Besides, the direct observation of quasi-adiabatic temperature $T_{\text {qad }}^{ \pm}(B)$ reveals richer properties of a magnetic material which cannot be predicted from the equilibrium thermodynamics: '+'('-')-sign in the superscript of a function or a variable indicates that $B$ is increasing (decreasing). Let $T_{\text {fit }}^{ \pm}$be a theoretical estimation for $T_{\text {qad }}^{ \pm}$. It is presumed that correction factors to $T_{\text {ad }}^{ \pm}$, such as dissipation by nonequilibrium effects originating from irreversible motions of magnetic domains and from quasi-particle scattering upon phase transitions, are added up for $T_{\text {fit }}^{ \pm}(B)$ [22]. The non-equilibrium, irreversible correction to $T_{\mathrm{ad}}^{ \pm}$is denoted by $T_{\text {neq. }}^{ \pm}$A correction factor due to thermal conduction between the specimen and the thermal reservoir ( $i$. $e$. the mixing chamber of a dilution fridge) at a temperature $T_{\text {mix }}$ should also be considered. Formally, an eddy current perturbation, $T_{\text {eddy }}$, to $T_{\text {ad }}^{ \pm}$is added even if it is negligible in many systems, unless a pulsed magnet is used. Finally, the equation for $T_{\text {fit }}^{ \pm}(B)$ is written as

$$
\frac{T_{\mathrm{fit}}^{ \pm}(B)}{d B}=\frac{d T_{\mathrm{ad}}^{ \pm}}{d B} \mp \frac{1}{|r| C_{p}(T, B)} \int_{T_{\text {mix }}}^{T_{\mathrm{fit}}^{ \pm}} d T K(T, B) \pm \frac{d T_{\mathrm{neq}}^{ \pm}}{d B} \pm \frac{d T_{\mathrm{eddy}}}{d B}
$$

where $K$ is a thermal conduction coefficient between the sample and the reservoir, and $r$ is the field sweep-rate. In Eq. (6), plus-minus signs are in the same order.
The ansatzes for $d T_{\mathrm{ad}}^{ \pm} / d B$ and $d T_{\mathrm{qad}}^{ \pm} / d B$ are as follows,

$$
\begin{aligned}
\frac{d T_{\mathrm{ad}}^{ \pm}}{d B}= & a^{ \pm} \operatorname{sech}^{2}\left\{w_{1}^{ \pm}\left(B-B_{\mathrm{c}}^{ \pm}\right)\right\} \\
& +b^{ \pm}\left[\tanh \left\{w_{2}^{ \pm}\left(B-B_{\mathrm{c}}^{ \pm}\right)\right\}+c\right],
\end{aligned}
$$




$$
\frac{d T_{\text {neq }}^{ \pm}}{d B}=\sum_{l=1}^{2} h_{l}^{ \pm} \operatorname{sech}^{2}\left\{\delta_{l}^{ \pm}\left(B-B_{l}^{ \pm}\right)\right\},
$$

where $a^{ \pm}, b^{ \pm}, c^{ \pm}, w_{1}^{ \pm}$, and $w_{2}^{ \pm}$are adjustable parameters to mimic $T_{\mathrm{ad}}^{ \pm}(B)$ as read from $S(T, B)$. When integrated over $B$, the first term of Eq. (7) depicts a stepwise change of $T_{\mathrm{ad}}^{ \pm}$in the vicinity of a critical field, $B_{\mathrm{c}}^{ \pm}$, whose sharpness is controlled by $w_{1}^{ \pm}$. The second term has been devised to approximate the equilibrium ansatz in a wider range decribing a rather smooth MCE. The hyperbolic functions in Eq. (8) are borrowed to depict nonequilibrium temperature variations around $B_{l}^{ \pm}$, which are presumed to originate from magnetic domain motions and/or quasi-particle scatterings at a phase boundary [22]. In this phenomenological model, the magnitude of heating is controlled by $h_{l}^{ \pm}$, and the degree of focusing of the heat is tuned by $\delta_{l}^{ \pm}$. It has to be emphasized that Eqs. (7) and (8) are ansatzes $i$. e. trial functions. Compared with our previous model [22], Eq. (6) is nothing but a rather sophisticated expression with different nomenclature and formal inclusion of the eddy current effect. Eq. (6) is a starting equation to understand practical magnetocaloric phenomena which can be written by anyone who agrees to add corrections to Eq. (2).

In Section IV, two examples will be dealt with using the model developed in this section. For instance, instead of applying Eq. (5), we exhibit solutions to Eq. (6) by setting $a^{ \pm}=0$ and $w_{2}^{ \pm}=0$ in Eq. (7). Also, $h_{l}^{ \pm}$ is set to zero in Eq. (8) because we do not expect nonequilibrium phenomena related to magnetic domains or distinct phase boundaries down to very low temperature in a paramagnet. By doing so, we reduce Eq. (6) to $d T_{\text {ad }}^{ \pm}(B) / d B=b^{ \pm} c$. Then, $T_{\text {ad }}^{ \pm}(B)=b^{ \pm} c\left(B+B_{\text {rem }}\right)$, where $b^{ \pm} c \times B_{\text {rem }}$ becomes the $y$-intersection in Fig. 2 below.

Meanwhile, the irreversible MCE observed in $\mathrm{Ce}_{0.5} \mathrm{La}_{0.5} \mathrm{~B}_{6}$ will be analyzed with nonzero $d T_{\text {neq }}^{ \pm} / d B$. The shape of an isentropic path is rather simple in this material compared to those found from $\mathrm{Ce}_{1-x} \mathrm{La}_{x} \mathrm{~B}_{6}$ with different $x$ [22]. For practical calculations, $T_{\mathrm{ad}}^{ \pm}(B)$ was found by fitting it to an isentropic contour extracted from $S(T, B)$. This means that we know $a^{ \pm}, b^{ \pm}, c^{ \pm}, w_{1}^{ \pm}$, and $w_{2}^{ \pm}$from the least-square fitting. Once the first term in Eq. (6), $T_{\mathrm{ad}}^{ \pm}(B)$, is approximated, the second and the third terms are added. Then, Eq. (6) was iteratively integrated by using the $4^{\text {th }}$-order Runge-Kutta method with different set of parameters $\left\{h_{l}^{ \pm}, \delta_{l}^{ \pm}, B_{l}^{ \pm}\right\}$until the final least-square solution was found.

\section{MATERIALS AND METHODS}

\section{A. Material Preparation}

$$
\text { 1. } \mathrm{YbPt}_{2} \mathrm{Sn}
$$

To synthesize $\mathrm{YbPt}_{2} \mathrm{Sn}$ in the form of cylindrical ingot, both an arc-melting furnace and an RF induction furnace were used. The melting temperature of $\mathrm{Yb}$ is about $1100 \mathrm{~K}$, that of $\mathrm{Pt}$ is about $2000 \mathrm{~K}$, and that of $\mathrm{Sn}$ is about $500 \mathrm{~K}$. Because of largely different melting points of constituent elements, a button-shaped YbSn precursor was first synthesized inside the arc-furnace which was filled with high purity Ar gas. The button and Pt chunks were put together inside the arc-furnace and forced to react near the melting point of Pt. Through the two-step reaction process, a few percent loss of $\mathrm{Yb}$ was observed because the boiling temperature of $\mathrm{Yb}$ is around $1470 \mathrm{~K}$ while the melting point of $\mathrm{Pt}$ is about $2000 \mathrm{~K}$. The $\mathrm{Yb}$ loss was therefore compensated by adding about $10 \%$ more $\mathrm{Yb}$ compared to the stoichiometric amount from the beginning. For the shaping, the as-grown and rather irregularly formed bulk $\mathrm{YbPt}_{2} \mathrm{Sn}$ was placed in an $\mathrm{RF}$ induction furnace and cast into an ingot pillar.

The powder X-ray diffraction pattern was analyzed, and we have confirmed that the space group of crystalline $\mathrm{YbPt}_{2} \mathrm{Sn}$ was $P 6_{3} / m m c$, with lattices parameters $a=$ $4.4862 \AA$ and $c=8.8881 \AA$. Say, $\mathrm{YbPt}_{2} \mathrm{Sn}$ crystallizes with a hexagonal $\mathrm{ZrPt}_{2} \mathrm{Al}$ type structure. The crystal structure is reproduced in Fig. 1(a) [25]. Small pieces of $\mathrm{YbPt}_{2} \mathrm{Sn}$ were used to investigate electric, magnetic, and thermodynamic properties. About $95 \%$ of the as-grown sample was $\mathrm{YbPt}_{2} \mathrm{Sn}$, while small islands of $\mathrm{Yb}_{2} \mathrm{Pt}_{48} \mathrm{Sn}_{40}$ were observed as a result of the energy-dispersive $\mathrm{X}$-ray analysis (EDXA). More details about the material growth can be found in reference [26].

\section{La substituted $\mathrm{CeB}_{6}$}

La substituted $\mathrm{CeB}_{6}, \mathrm{Ce}_{0.5} \mathrm{La}_{0.5} \mathrm{~B}_{6}$, was prepared by using the floating-zone technique [27]. First, the powder precursor was synthesized by using chemical reactions, $\mathrm{CeO}_{2}+8 \mathrm{~B} \rightarrow \mathrm{CeB}_{6}+2 \mathrm{BO}$ and $\mathrm{La}_{2} \mathrm{O}_{3}+15 \mathrm{~B} \rightarrow 2 \mathrm{LaB}_{6}+3 \mathrm{BO}$, around $1900 \mathrm{~K}$. The powder form of $\mathrm{Ce}_{1-x} \mathrm{La}_{x} \mathrm{~B}_{6}$ was pelletized into a $60 \mathrm{~mm}$ long cylinder of $8 \mathrm{~mm}$ in diameter. A customized crucible-free inductive floating zone furnace was used to grow a single crystal after the pellet was sintered at $2000 \mathrm{~K}$. An optical spectral analysis revealed that the impurity concentration was below $10^{-3}$ mass $\%$. For the measurement of thermodynamic properties, moderate size of pieces of single crystals were cut from the large specimen explained above, which was prepared for a magnetic neutron scattering experiment.

Single crystals with various La content were characterized by analyzing X-ray and neutron diffraction patterns, and it has been verified that the structure of $\mathrm{Ce}_{1-x} \mathrm{La}_{x} \mathrm{~B}_{6}$ belongs to the cubic $P m \overline{3} m$ space group. Equivalently, the material crystalizes into the $\mathrm{CsCl}$ type cubic structure. The lattice parameter $a$, determined by $\mathrm{X}$-ray or neutron diffraction experiment on $\mathrm{Ce}_{0.5} \mathrm{La}_{0.5} \mathrm{~B}_{6}$, was about $4.1466 \AA$. More precisely, it is known that $a=4.13899 \AA$ for $\mathrm{CeB}_{6}$ and $a=4.15553 \AA$ for $\mathrm{LaB}_{6}$ at $300 \mathrm{~K}$ [28-30]. The detailed structure is shown in Fig. 1(b) [25]. A rare-earth element is surrounded by eight boron-octahedra.

Although the lattice looks simple, its spin and orbital structure encoded in the multipolar, pseudospin state is notoriously complicated [22, 31-35]. Especially, the magnetic field dependent anisotropy of multipolar phases is pronounced, and an alignment of a crystallographic axis towards a certain direction of magnetic field is important. In our work, X-ray Laue backscattering patterns 

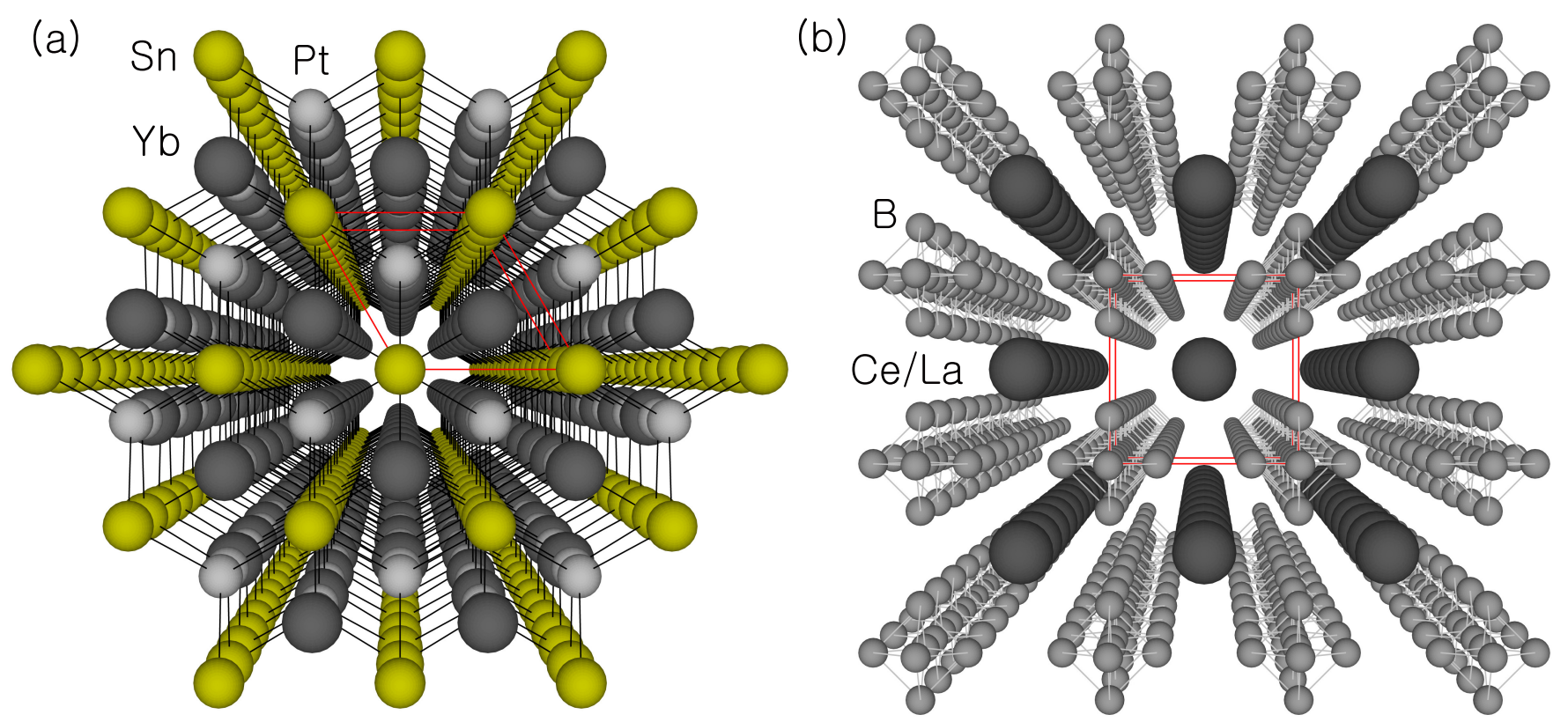

FIG. 1. (color) (a) Crystal structure of $\mathrm{YbPt}_{2} \mathrm{Sn}$ is shown. A conventional unit cell for $P 6_{3} / m m c$ space group is denoted by red lines. Lattice parameters are $a=4.4862 \AA$ and $c=8.8881 \AA$. Gold-colored spheres mark positions of Sn, spheres with light-gray color mark Pt atoms, dark-gray spheres mark Yb atoms. Nearest Pt-Sn pairs are indicated by thin black lines. These pairs consist of Pt-Sn atomic layers spaced by planes of $\mathrm{Yb}$ atoms. (b) Crystal structure of La-substituted CeB 6 is presented. A conventional unit cell corresponding to the $\operatorname{Pm} \overline{3} m$ cubic structure is drawn by red lines at the center of the figure. The lattice parameter is $a \approx 4.147 \AA$. A rare-earth atom is surrounded by boron octahedra placed at the corners of the unit cell. Crystallographic $\hat{c}$-axes in both figures are coincident with the paper normal.

were used to determine crystallographic orientations.

\section{B. Measurements}

\section{Specific heat capacity and entropy}

The heat capacity of $\mathrm{YbPt}_{2} \mathrm{Sn}$ and $\mathrm{Ce}_{0.5} \mathrm{La}_{0.5} \mathrm{~B}_{6}$ was measured by using the compensated heat pulse method in a ${ }^{3} \mathrm{He} /{ }^{4} \mathrm{He}$ dilution fridge $[12,22,36]$. For $\mathrm{YbPt}_{2} \mathrm{Sn}, C_{p}$ was investigated in a temperature range from 0.05 to $4 \mathrm{~K}$, and the external magnetic field was applied up to $7 \mathrm{~T}$. The heat capacity of $\mathrm{Ce}_{0.5} \mathrm{La}_{0.5} \mathrm{~B}_{6}$ was measured over the same temperature range with the external magnetic field up to $4 \mathrm{~T}$. In the figures below, the specific heat capacity is not shown for the sake of simple data representation. Instead, the entropy $S(T, B)=\int\left(C_{p} / T\right) d T$, will be discussed intensively in conjunction with the MCE.

\section{Magnetocaloric effect}

A custom adiabatic demagnetization refrigerator (ADR) was built to examine metallic $\mathrm{YbPt}_{2} \mathrm{Sn}$ as a solid state cooling agent. The ADR stage was attached below the $1 \mathrm{~K}$ pot of the normal ${ }^{4} \mathrm{He}$-cryostat. An experimental stage made out of brass, where we can mount a specimen, was separated from the $1 \mathrm{~K}$ pot (the thermal reservoir) by a pair of insulating straws. Below the experimental stage, $10 \mathrm{~g}$ of $\mathrm{YbPt}_{2} \mathrm{Sn}$ ingot was attached through a pair of long brass rods of $1 \mathrm{~mm}$ diameter. A push-pull mechanical heat switch was installed whose tip was anchored to the reservoir. Once the switch was closed, the tip touched the experimental stage and thermal equilibrium was achieved between the thermal reservoir, $30 \mathrm{~g}$ of brass structure, and $10 \mathrm{~g}$ of $\mathrm{YbPt}_{2} \mathrm{Sn}$. Temperatures were estimated by converting the resistances of a $\mathrm{RuO}_{2}$ thick film into a temperature. The location of the $\mathrm{YbPt}_{2} \mathrm{Sn}$ pillar was designed to be at the center of the superconducting magnet equipped in the liquid ${ }^{4} \mathrm{He}$ Dewar.

At the beginning of the operation, external field was applied with the heat switch closed to suppress the entropy isothermally. A quasi-adiabatic state was made by opening the heat switch. Then, the temperature variation of the $\mathrm{YbPt}_{2} \mathrm{Sn}$ ingot was monitored as the external field was removed or applied.

The same apparatus for the compensated heat pulse method was used to observe the MCE in $\mathrm{Ce}_{0.5} \mathrm{La}_{0.5} \mathrm{~B}_{6}$. An L-shaped sample platform made out of low-impurity silver was supported by thin nylon threads at the center of a silver ring of $3 \mathrm{~cm}$ diameter. Because we did not install a heat switch, it took about 6 hours to thermally equilibrate the sample and the mixing chamber. Although the quality of the insulation was not as good as the degree of the insulation realized in the home-made ADR, it was good enough to investigate essential features of the MCE by applying the model developed in Section II. 


\section{RESULTS AND DISCUSSION}

A. Reversible MCE: Adiabatic demagnetization cooling with a novel metallic paramagnet $\mathrm{YbPt}_{2} \mathrm{Sn}$

The temperature dependent $C_{p}$ was investigated for $0.05 \mathrm{~K}<T<4 \mathrm{~K}$ at different values of $B$ ranging from 0 to $7 \mathrm{~T}$ with a $0.5 \mathrm{~T}$ interval. For each value of $B$, $C_{p}(T)$ was measured at roughly two hundred different temperatures. The nuclear contribution to $C_{p}$ was subtracted because the MCE is dominated by the $4 f$ electron wave functions. The eight-fold degenerate energy levels of free- $\mathrm{Yb}^{3+}$ ions, with total angular momentum quantum number $J=7 / 2$, split into four Kramers doublets under the influence of the hexagonal crystal electric field (CEF): refer to Fig. 1(a). With a group-theoretical consideration [37], it is summarized as, $D^{\left(\frac{7}{2}\right)} \downarrow D_{6 h} \rightarrow$ $\Gamma_{7} \oplus 2 \Gamma_{8} \oplus \Gamma_{9}$. Wave functions corresponding to $\Gamma_{7}$ representation are $\left| \pm \frac{1}{2}\right\rangle$, wave functions corresponding to $\Gamma_{8}$ representation are $\alpha\left| \pm \frac{7}{2}\right\rangle+\beta\left|\mp \frac{5}{2}\right\rangle$ and $\beta\left| \pm \frac{7}{2}\right\rangle-\alpha\left|\mp \frac{5}{2}\right\rangle$. Physical properties such as neutron spectra or magnetization at higher temperatures are additionally needed to determine $\alpha$ and $\beta$. Wave functions $\left| \pm \frac{3}{2}\right\rangle$ are the basis of the $\Gamma_{9}$ representation. The saturated magnetization at low temperatures was well explained by the $\Gamma_{7}$ doublet. In consequence, $\left| \pm \frac{1}{2}\right\rangle$ is the ground state wave function, and $\mathrm{YbPt}_{2} \mathrm{Sn}$ can be regarded as a metallic paramagnet below approximately $4 \mathrm{~K}[12]$.

Fig. 2 presents a colored contour plot of $4 f$ electronic entropy $S_{4 f}(T, B)$ of $\mathrm{YbPt}_{2} \mathrm{Sn}$. As discussed in Section II, only $C_{p}(T, B)$ was enough to calculate $T_{\text {ad }}(B)^{ \pm}$. Instead of using the analytic result (Eq. (5)) and its realistic modification, which assigns a finite temperature at $B_{\mathrm{f}}=0$, Eq. (6) has been solved and experimental results were fitted to prove the validity of our practical approach.

To conduct an experiment, the normal operating procedure of an ADR was followed. First, we suppress entropy by applying $B=4 \mathrm{~T}$ at $T=1.75 \mathrm{~K}$. Second, we opened the heat switch to thermally isolate $\mathrm{YbPt}_{2} \mathrm{Sn}$. Lastly, we increased $B$ with $r=-0.1 \mathrm{~T} / \mathrm{min}$. The data were captured each second. In the figure, 80 out of 2400 data points are overlapped on the countour plot (white lefttriangles). After 3 hours, $T_{\text {qad }}^{-}(0)$ increased from $0.23 \mathrm{~K}$ to $0.26 \mathrm{~K}$. From this coordinate in the $B-T$ plane, $B$ is increased to $2 \mathrm{~T}$ with $r=0.05 \mathrm{~T} / \mathrm{min}$. The result is marked by with right-triangles in Fig. 2. In this sweep-up procedure, 40 out of 4800 data points are shown.

For the model calculation, $T_{\text {ad }}^{ \pm}(B)$ was numerically estimated from $S_{4 f}(T, B)$. Relevant parameters of materials which connect the reservoir and the ingot were collected to evaluate $K(T, B)$. The non-equilibrium, irreversible feature was absent in this paramagnetic system. Subsequent magnetic cooling after 1 hour of intermission at $(B, T)=(2 \mathrm{~T}, 1.26 \mathrm{~K})$ exhibited an almost reversible $T_{\text {qad }}^{ \pm}(B)[12]$. The power dissipation by the eddy current was discarded from the calculations as it is perturbatively small (see Appendix). The red solid line behind the white left-triangles designates $T_{\text {fit }}^{-}(B)$ and well explains the experimental data. Similarly, the black solid line behind the white right-triangle designates $T_{\text {fit }}^{+}(B)$ with two times slower sweep-rate than that of the cooling process.

Obviously, isentropic paths are straight lines imply-

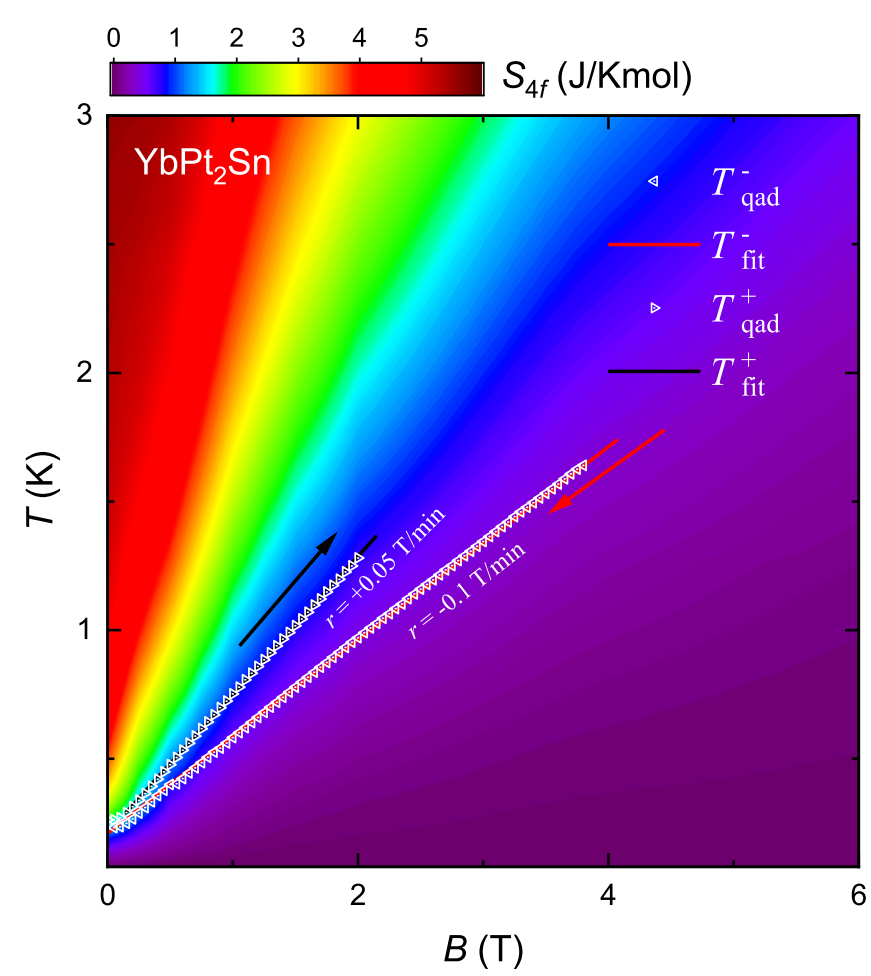

FIG. 2. (color) The background color plot shows the $4 f$ electronic entropy of $\mathrm{YbPt}_{2} \mathrm{Sn}$. Although it is not shown, $S_{4 f}(4 K, 0) \approx R \ln 2$ which implies the ground state is a doublet as explained in the text. Also note that the scale bar is stretched up to $R \ln 2$. White left-triangles present $T_{\text {qad }}^{-}$with $r=-0.1 \mathrm{~T} / \mathrm{min}$ (see the red arrow). The final temperature, $T_{\text {qad }}^{-}(0)$ was $0.23 \mathrm{~K}$. The red solid line behind the left-triangles is drawn based on the solution to Eq. (6), $T_{\mathrm{fit}}^{-}(B)$, with the initial condition $\left(B_{\mathrm{i}}, T_{\mathrm{i}}\right)=(4 \mathrm{~T}, 1.75 \mathrm{~K})$. Similarly, white righttriangles designate $T_{\text {qad }}^{+}(B)$ with $r=+0.05 \mathrm{~T} / \mathrm{min}$ after 3 hours of the first sweep down. Behind the experimental data, $T_{\text {fit }}^{+}(B)$ which is the solution to Eq. (6) with the initial condition $\left(B_{\mathrm{i}}, T_{\mathrm{i}}\right)=(0,0.26 \mathrm{~K})$ is shown by the black solid line. The same raw data for specific heat capacity in Ref. [12] were used to create the color-coded plot, while experimental points for the MCE are original in this article.

ing that this material is a good candidate for a solid state magnetic cooling material below $4 \mathrm{~K}$. Besides, there are two more important implications. First, ever since the discovery of paramagnetic salts and garnets, supplementing the low thermal conductivity of these materials in combination with highly conducting metal structures has been the most difficult task in building an $\operatorname{ADR}[8,9,11,13]$. The metallic $\mathrm{YbPt}_{2} \mathrm{Sn}$ is the MCM which can be installed in an ADR without concerning material issues inherent to insulating paramagnet. Second, the base temperature of our home-made ADR is below $300 \mathrm{mK}$. This fact strongly suggests that one can build an alternative to the widespread ${ }^{3} \mathrm{He}$-cryostat which will be very rare in the near future because of a serious supply cutback of ${ }^{3} \mathrm{He}[47,48]$.

It seemed hard to defy the tendency that magnetic ordering temperature, $T_{\mathrm{m}}$, increases with material density, $d$. The black arrow stretching from the group of PM salts to the group of rare-earth (RE) intermetallic compounds in Fig. 3 represents such a trend. Since the lowest reach- 


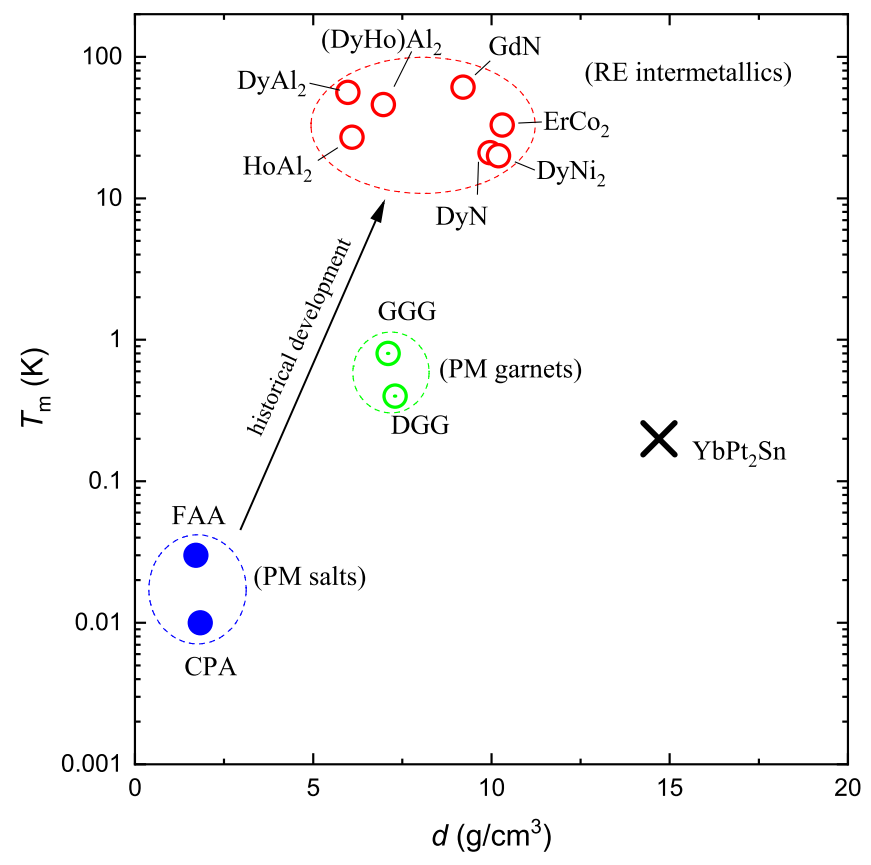

FIG. 3. (color) Magnetic transition temperatures, $T_{\mathrm{m}}$, of various magnetocaloric materials are arranged with their physical density, $d$. CPA is the abbreviation for chromium potassium alum, $\mathrm{CrK}\left(\mathrm{SO}_{4}\right)_{2} \cdot 12\left(\mathrm{H}_{2} \mathrm{O}\right)[6,13]$. FAA is the abbreviation for ferric ammonium alum, $\mathrm{FeNH}_{4}\left(\mathrm{SO}_{4}\right)_{2} \cdot 12\left(\mathrm{H}_{2} \mathrm{O}\right)[13,38]$. Inside the area bounded by the green dashed line, representative PM garnets, gadolinium gallium garnet (GGG, $\left.\mathrm{Gd}_{3} \mathrm{Ga}_{5} \mathrm{O}_{12}\right)[7,13,39]$ and dysprosium gallium garnet (DGG, $\left.\mathrm{Dy}_{3} \mathrm{Ga}_{5} \mathrm{O}_{12}\right)[10,13,16,40]$ are positioned. Inside the area bounded by the red dashed line are $\mathrm{RE}$ intermetallic compounds such as $\mathrm{HoAl}_{2}$ [41], $\mathrm{DyAl}_{2}$ [42], (DyHo) $\mathrm{Al}_{2}$ [43], GdN [44], ErCo2 [15, 43], DyNi 2 [45], and DyN [44]. The arrow with the black solid line designates a rough historical direction for the development of MCMs. It reveals a tendency of increasing $T_{\mathrm{m}}$ with $d$. To note, there are many more $\mathrm{RE}$ intermetallics outside the graph $[43,46]$. In contrast to this seemingly reasonable observation, we note the location of $\mathrm{YbPt}_{2} \mathrm{Sn}$ (black $\times$-mark) largely deviates from the historical tendency.

able temperature is roughly equal to $T_{\mathrm{m}}$, it was difficult to find a metallic MCM with $T_{\mathrm{m}}<0.3 \mathrm{~K}$ along the arrow. In this sense, $\mathrm{YbPt}_{2} \mathrm{Sn}(\times$-mark in Fig. 3$)$ is the first metallic paramagnet suitable for cryogenic cooling from liquid ${ }^{4} \mathrm{He}$ temperature down to $0.2 \mathrm{~K}$. On the other hand, the primary reason why the location of $\mathrm{YbPt}_{2} \mathrm{Sn}$ largely departs from the historical path is still unknown. We hope to find a group of materials around the $\times$-mark so that we can conduct a comparative study.

Although we have discussed many things implied by the discovery of $\mathrm{YbPt}_{2} \mathrm{Sn}$, it must be reminded that this material is a metal which shows an almost linear and reversible MCE [12].

\section{B. Irreversible MCE: Field dependent heating in a complex-phase material $\mathrm{Ce}_{0.5} \mathrm{La}_{0.5} \mathrm{~B}_{6}$}

There are six-fold degenerate wave-functions for a bare $\mathrm{Ce}^{3+}$ ion with total angular moment quantum number $J=5 / 2$, and these are split by the cubic CEF pertur-

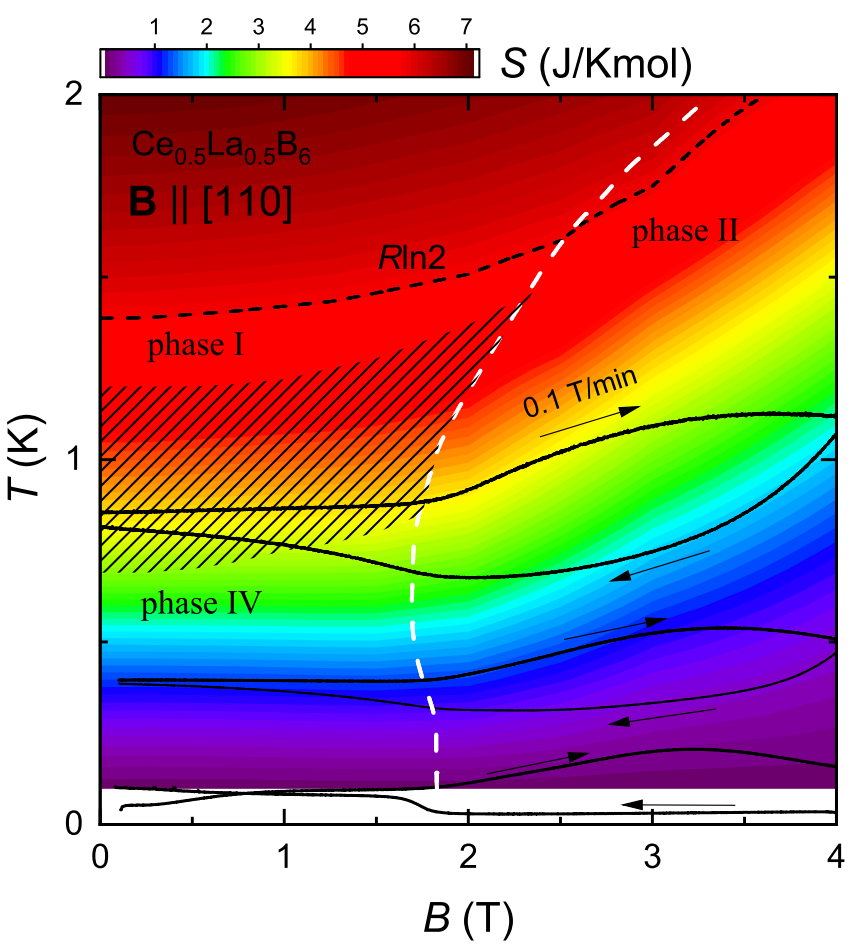

FIG. 4. (color) Black lines indicate $T_{\text {qad }}^{ \pm}(B)$ from $\mathrm{Ce}_{0.5} \mathrm{La}_{0.5} \mathrm{~B}_{6}$ $(|r|=0.1 \mathrm{~T} / \mathrm{min})$. The white dashed line denotes the IV $\rightarrow \mathrm{II}$ phase boundary and the black hatched area stands for the broad transition between phase I and phase IV. A contour at the value $R \ln 2$ is denoted by the black dashed line. For the lowest lying $T_{\text {qad }}^{ \pm}(B), T_{\text {mix }}$ was $0.2 \mathrm{~K}$. For $T_{\text {qad }}^{ \pm}(B)$ in the middle, $T_{\text {mix }}$ was $0.4 \mathrm{~K}$. For $T_{\text {qad }}^{ \pm}(B)$ at the top, $T_{\text {mix }}$ was $0.8 \mathrm{~K}$. Approximately below $0.05 \mathrm{~K}$, measurement of the specific heat capacity was not reliable, and the background contour plot below $0.08 \mathrm{~K}$ is absent. The same specific heat capacity data in Ref. [22] were used to create the color-coded plot. On the other hand, phase boundaries are original in this article.

bation which is apparent from Fig. 1(b). In terms of group theory, the degeneracy is lifted as $D^{\left(\frac{5}{2}\right)} \downarrow O_{h} \rightarrow$ $\Gamma_{7} \oplus \Gamma_{8}$. Early Raman and neutron scattering experiments confirmed that the $\Gamma_{8}$ quartet lies $\Delta E=46 \mathrm{meV}$ $\left(\Delta E / k_{\mathrm{B}}=534 \mathrm{~K}\right)$ below the $\Gamma_{7}$ doublet [49]. Therefore, low temperature physical properties are manifested by pseudospin states spanned by the $\Gamma_{8}$-quartet. A group of wave functions for $\Gamma_{8}$ representations consists of $\left| \pm \frac{1}{2}\right\rangle$ and $\sqrt{\frac{5}{6}}\left| \pm \frac{5}{2}\right\rangle+\sqrt{\frac{1}{6}}\left|\mp \frac{3}{2}\right\rangle$ Kramers doublets.

There exist dipolar, quadrupolar, and octupolar phases in $\mathrm{Ce}_{1-x} \mathrm{La}_{x} \mathrm{~B}_{6}$ depending on $x, T$, and $\mathbf{B}$. It is customary to name the paramagnetic phase as phase I, the antiferro-quadrupolar (AFQ) phase as phase II [50-52], and the antiferromagnetic (AFM) phase as phase III [5355]. Phase IV is allegedly an antiferro-octupolar (AFO) phase [56-60]. Phase diagrams are also strongly dependent on the orientation of $\mathbf{B}$ relative to the crystal axes and it is known that the most diverse phases appear when B \| [110]. Precise but complicated phase diagrams of $\mathrm{Ce}_{1-x} \mathrm{La}_{x} \mathrm{~B}_{6}$ were obtained by combined analysis of magnetic neutron scattering experiments, specific heat capacity, and quasi-adiabatic MCE for B $\|$ [110] [22]. In this article, $\mathrm{Ce}_{0.5} \mathrm{La}_{0.5} \mathrm{~B}_{6}$ is analyzed with additional data and 


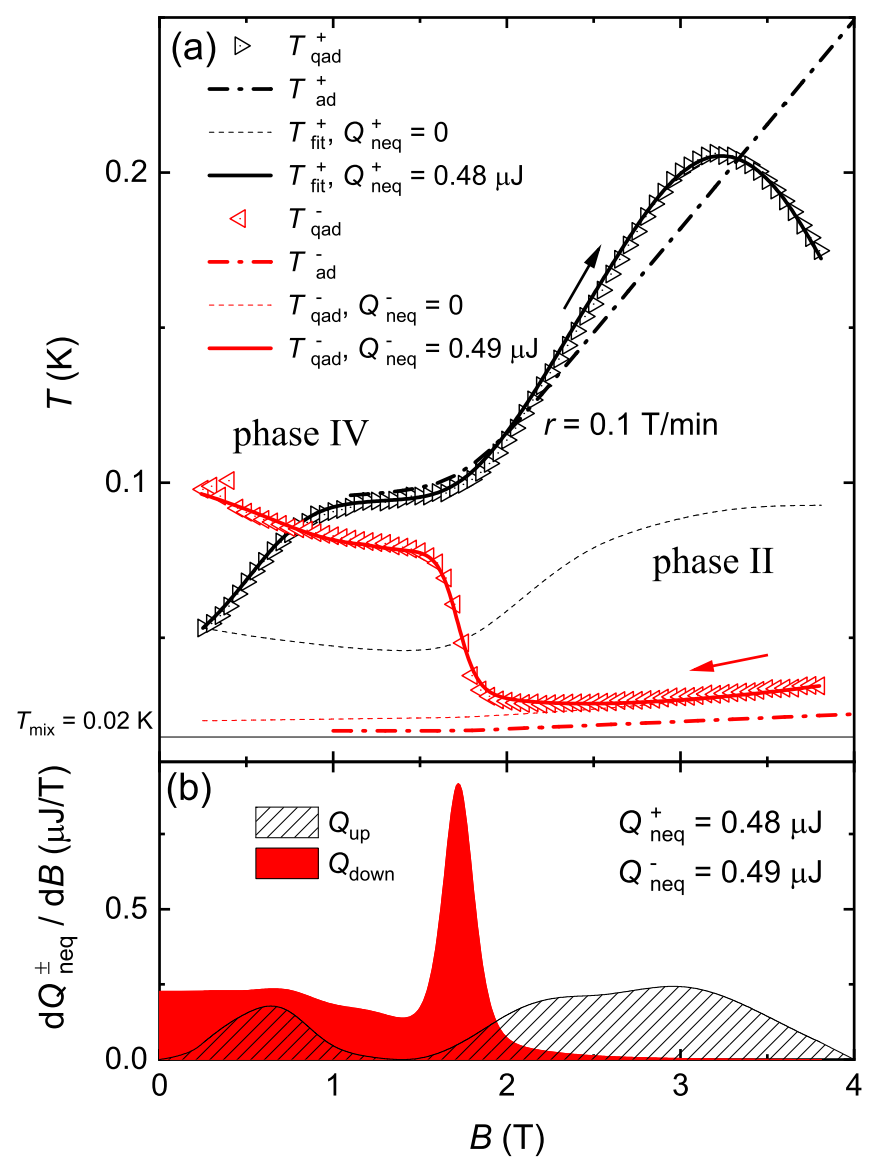

FIG. 5. (color) (a) Quasi-adiabatic, non-equilibrium MCE from $\mathrm{Ce}_{0.5} \mathrm{La}_{0.5} \mathrm{~B}_{6}$ with $T_{\text {mix }}=0.02 \mathrm{~K}$ is analyzed in detail. Black right-triangles show $T_{\text {qad }}^{+}(B) . \quad T_{\text {ad }}^{+}(B)$ is approximated by black dash-dotted line. Black dashed line denotes a solution to Eq. (6), $T_{\text {fit }}^{+}(B)$, without the correction term, $d T_{\text {neq }}^{+} / d B$. Black solid line is the final solution to Eq. (6) with all the non-equilibrium correction terms. Similarly, red left-triangles show $T_{\text {qad }}^{-}(B)$. Red dashdotted line is $T_{\text {ad }}^{-}(B)$. The red dashed line is $T_{\text {fit }}^{-}$without non-equilibrium corrections. Red solid line represents the final solution to Eq. (6) with all the correction terms. Since $C_{p}(T, B)$ was unavailable roughly below $0.05 \mathrm{~K}$, it was presumed $C_{p}(T, B)=C_{p}(0.08 \mathrm{~K}, 0)$ below $0.08 \mathrm{~K}$. (b) Irreversible heating curves which were adjusted to fit $T_{\text {qad }}^{ \pm}(B)$ are presented. Sets of parameters $\left\{a^{+}=0, b^{+}=\right.$ $0.032 \mathrm{~K} / \mathrm{T}, c^{+}=1.1, w_{2}^{+}=3.75 \mathrm{~T}^{-1}, h_{1}^{+}=0.146 \mathrm{~K} / \mathrm{T}, h_{2}^{+}=$ $0.087 \mathrm{~K} / \mathrm{T}, \delta_{1}^{+}=0.92 \mathrm{~T}^{-1}, \delta_{2}^{+}=2.125 \mathrm{~T}^{-1}, B_{1}^{+}=$ $\left.2.975 \mathrm{~T}, B_{2}^{+}=0.57 \mathrm{~T}, B_{c}^{+}=1.75 \mathrm{~T}\right\}$ and $\left\{a^{-}=0, b^{-}=\right.$ $0.0012 \mathrm{~K} / \mathrm{T}, c^{-}=1, w_{2}^{-}=10 \mathrm{~T}^{-1}, h_{1}^{-}=0.2012 \mathrm{~K} / \mathrm{T}, h_{2}^{-}=$ $0.068 \mathrm{~K} / \mathrm{T}, \delta_{1}^{-}=8.75 \mathrm{~T}^{-1}, \delta_{2}^{-}=1 \mathrm{~T}^{-1}, B_{1}^{-}=1.72 \mathrm{~T}, B_{2}^{-}=$ $\left.0.45 \mathrm{~T}, B_{c}^{-}=B_{c}^{+}=1.75 \mathrm{~T}\right\}$ were used.

with much more detail compared to our previous work.

The equilibrium thermodynamic characteristics of $\mathrm{Ce}_{0.5} \mathrm{La}_{0.5} \mathrm{~B}_{6}$ are well implied by the color-coded contour plot in Fig. 4. Inside phase II, $T_{\mathrm{ad}}^{+}(B)$ increases because AFM order is induced and the pseudospin state of phase II is stabilized. On the other hand, phase IV is almost insensitive to $B$. These features are properly captured in the analytic function by adjusting parameters in Eq. (7). The eddy current heating was negligible (refer to Appendix). The thermal conduction coefficient $K \approx\left(1.2 \times 10^{-7}\right) T^{1.2} \mathrm{~W} / \mathrm{K}$ was found below $4 \mathrm{~K}$ and the field dependence of $K$ was negligible up to $4 \mathrm{~T}$.

In Fig. $4, T_{\text {qad }}^{ \pm}(B)$ form loops which are indicative of heat conduction between the specimen and the mixing chamber at the temperature $T_{\text {mix }}$. Despite this, we could see that $T_{\text {qad }}^{+}(B)$ follows $T_{\text {ad }}^{+}(B)$ in a narrow range near the phase transition. The inflection points were taken as transition points from phase IV to phase II. The phase boundary (white dashed line) was interpolated based on similar inflection points found by conducting more magnetocaloric sweeps and based on transition temperatures determined by entropy-balancing near specific heat anomalies [22]. The IV $\rightarrow$ II transition looks like a disorder to order transition as $T_{\text {qad }}^{+}(B)$ increases across the transition. On the contrary, the lowest curve in Fig. 4 shows a distinct II $\rightarrow$ IV transition accompanied by a step-like increase in $T_{\text {qad }}^{-}$, implying that phase IV is more ordered than phase II. These are incompatible interpretations regarding the question of which phase is more ordered.

All the correction terms except the eddy current term in Eq. (6) need to be considered to settle the contradiction. Fig. 5(a) exhibits $T_{\text {qad }}^{ \pm}(B)$ (symbols) with a magnified temperature scale. Dash-dotted lines present $T_{\text {ad }}^{+}(B)$, which is extracted from $S(T, B)$. Non-equilibrium correction terms are visualized in Fig. $5(\mathrm{~b}), d T_{\text {neq }}^{ \pm} / d B(B)=$ $\frac{1}{C_{p}(T, B)} d Q_{\text {neq }}^{ \pm} / d B$. With optimized sets of parameters, $T_{\text {qad }}^{ \pm}$is nicely approximated by $T_{\text {fit }}^{ \pm}$as described by the black and red solid lines.

It is generally true that moving AFM domains with different orientations experience friction at the interfaces and heat could be released. In phase II, the AFQ order parameter coexists with the AFM order parameter which further stabilizes the pseudospin state with increasing $B$. Thus the substantial amount of $Q_{\text {neq }}^{+}$in phase II (above $2 \mathrm{~T}$ ) is indicative of heat expelled during the AFM domain alignment. The fact that $Q_{\text {neq }}^{-}$is much smaller than $Q_{\text {neq }}^{+}$in phase II supports the idea that domains were well aligned during the first sweep. A similar hysteresis of the AFM domain configuration around the region where $Q_{\text {neq }}^{+}$ is substantial was also confirmed by a nuetron scattering experiment [33]. On the other hand, it is still unresolved why heat energy is given off from phase IV regardless of the sweep direction. So far, it has been known that the AFO order constitutes the primary phase which coexists with the secondary AFM order, but it is ambiguous whether one of these phases stabilizes the other. In a phenomenological aspect, we can only surmise that there will be too many misaligned magnetic domains to be aligned in phase IV.

Leaving detailed features behind, it should be emphasized that $T_{\text {qad }}^{-}(B)$ cannot be explained without strongly focused heating at the II $\rightarrow$ IV boundary. Our hypothesis is that if the system is driven into a disordered state, fieldinduced quasi-particle scattering at the phase boundary is strong enough to distort the equilibrium figure of the MCE.

A quasi-adiabatic, non-equilibrium analysis of the MCE observed from $\mathrm{Ce}_{0.5} \mathrm{La}_{0.5} \mathrm{~B}_{6}$ with $T_{\text {mix }} \approx 0.4 \mathrm{~K}$ is summarized in Fig. 6. In Fig. 6(a), $T_{\text {qad }}^{+}(B)$ (black right-triangles) and $T_{\text {qad }}^{-}(B)$ (red left-triangles), which were obtained with $|r|=0.015 \mathrm{~T} / \mathrm{min}$, are shown. Exactly the same protocol described in Section II and Fig. 5 was applied to find $T_{\mathrm{ad}}^{ \pm}$. At a glance, it looked as if the 

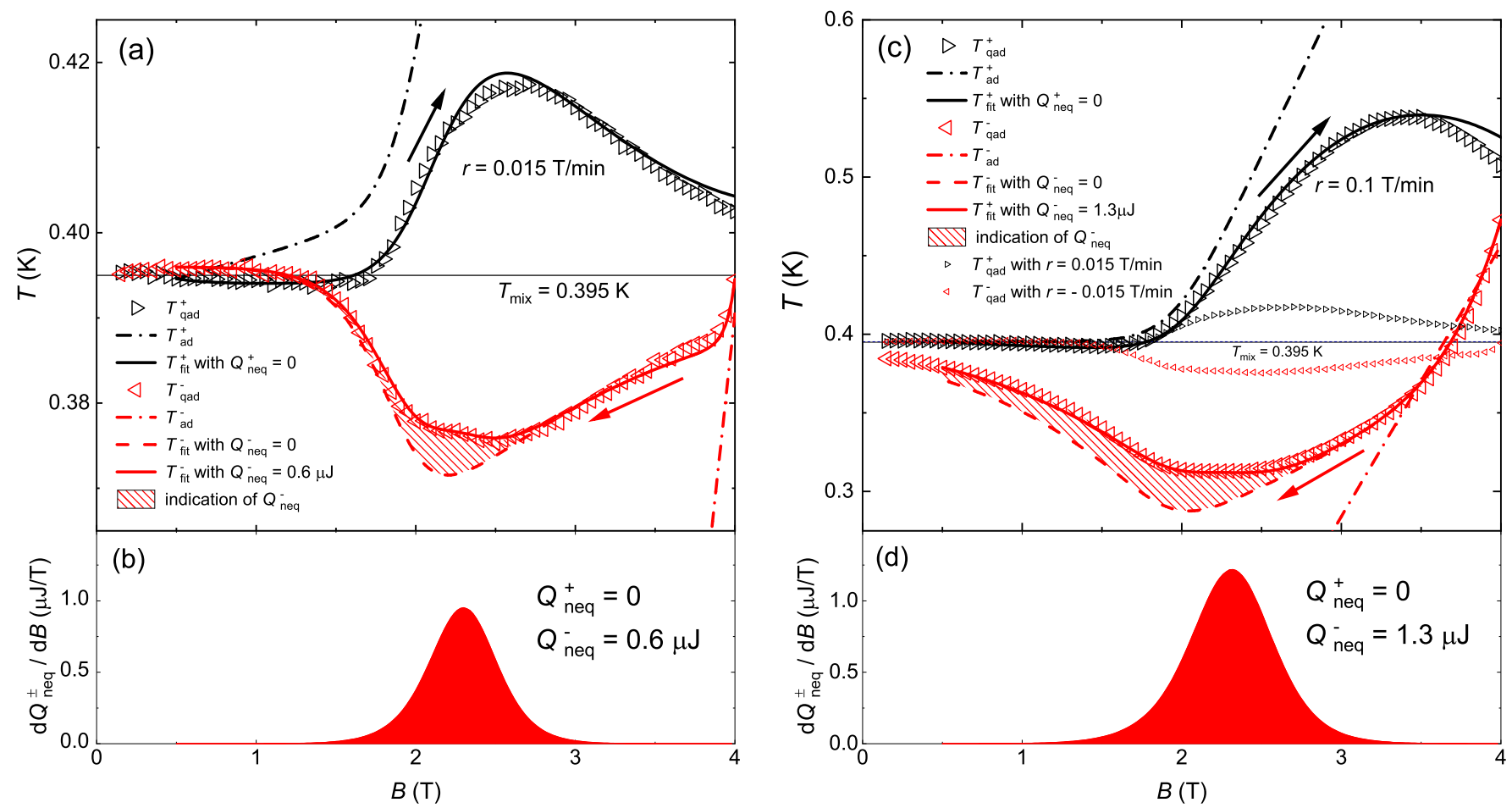

FIG. 6. (color) (a) The quasi-adiabatic, non-equilibrium MCE from $\mathrm{Ce}_{0.5} \mathrm{La}_{0.5} \mathrm{~B}_{6}$ with $T_{\operatorname{mix}} \approx 0.4 \mathrm{~K}$ and $|r|=0.015 \mathrm{~T} / \mathrm{min}$ is analyzed. Black right-triangles show $T_{\mathrm{qad}}^{+}(B) . T_{\mathrm{ad}}^{+}(B)$ is approximated by the black dash-dotted line. $T_{\text {fit }}^{+}(B)$ with zero heating (black solid line) well approximates $T_{\text {qad }}^{+}(B)$. Red left-triangles show $T_{\text {qad }}^{-}(B)$. The red dash-dotted line is $T_{\text {ad }}^{-}(B)$. The red dashed line is $T_{\text {fit }}^{-}$without non-equilibrium correction. The red solid line represents $T_{\text {fit }}^{-}$with $Q_{\text {neq }}^{-}$. The hatched area emphasizes the presence of $Q_{\text {neq. }}^{-}$(b) The field-dependent irreversible heating curves which are necessary to generate the black and red solid lines in (a) are displayed. (c) Data from the experiment which was conducted with an increased sweep-rate, $|r|=0.1 \mathrm{~T} / \mathrm{min}$, is presented. $T_{\text {qad }}^{ \pm}$with $|r|=0.015 \mathrm{~T} / \mathrm{min}$ is also shown for comparison. There is discrepancy between $T_{\mathrm{qad}}^{+}$and $T_{\mathrm{fit}}^{+}$above $3.5 \mathrm{~T}$ (see the text). (d) Field-dependent irreversible heating curves are displayed. Numerical fits in (a) were made by using the set of parameters $\left\{a^{+}=0, b^{+}=0.087 \mathrm{~K} / \mathrm{T}, c^{+}=1.06, w_{2}^{+}=3.5 \mathrm{~T}^{-1}, a^{-}=0, b^{-}=0.087 \mathrm{~K} / \mathrm{T}, c^{-}=1, w_{2}^{-}=4 \mathrm{~T}^{-1}, h_{1}^{-}=\right.$ $\left.0.0403 \mathrm{~K} / \mathrm{T}, \delta_{1}^{-}=3.5 \mathrm{~T}^{-1}, B_{1}^{-}=2.32 \mathrm{~T}, B_{c}^{ \pm}=1.98 \mathrm{~T}\right\}$. The set of parameters $\left\{a^{+}=0, b^{+}=0.1075 \mathrm{~K} / \mathrm{T}, c^{+}=1.015, w_{2}^{+}=\right.$ $\left.3.75 \mathrm{~T}^{-1}, a^{-}=0, b^{-}=0.0925, c^{-}=0.865, w_{2}^{-}=3.75 \mathrm{~T}^{-1}, h_{1}^{-}=0.067 \mathrm{~K} / \mathrm{T}, \delta_{1}^{-}=2.9 \mathrm{~T}^{-1}, B_{1}^{-}=2.35 \mathrm{~T}, B_{c}^{ \pm}=1.98 \mathrm{~T}\right\}$ was used for the best fit to the data in (b).

term $d T_{\text {neq }}^{ \pm} / d B$ would not be necessary to fit $T_{\text {qad }}^{ \pm}$because $r$ was thought to be slow enough to get rid of nonequilibrium effects. Although $T_{\text {fit }}^{+}(B)$ (black solid line) without $Q_{\text {neq }}^{+}$relatively well approximates $T_{\text {qad }}^{+}, T_{\text {fit }}^{-}$without $Q_{\text {neq }}^{-}$(red dashed line) certainly deviates from $T_{\text {qad }}^{-}$ in the vicinity of the II $\rightarrow$ IV boundary. The red hatchedregion in Fig. 6(a) stresses the very existence of $Q_{\text {neq }}^{-}(B)$ as shown in Fig. 6(b). The solution to Eq. (6) with finite $Q_{\text {neq }}^{-}(B)$ (red solid line in Fig. 6(a)) well reproduces $T_{\text {qad }}^{-}$.

In Fig. 6(c) and 6(d), the phenomenological analysis is repeated for the case with $|r|=0.1 \mathrm{~T} / \mathrm{min}$. For comparison, $T_{\text {qad }}^{ \pm}$measured with $|r|=0.015 \mathrm{~T} / \mathrm{min}$ is superposed. The red hatched area became much larger than the area shown in Fig. 6(a), implying that non-equilibrium effects upon entering phase IV of $\mathrm{Ce}_{0.5} \mathrm{La}_{0.5} \mathrm{~B}_{6}$ are stronger with faster field sweep-rate. To note, in Fig. 6(c), it seems $T_{\text {fit }}^{+}$well reproduces $T_{\text {qad }}^{+}$with $Q_{\text {neq }}^{+}=0$, but the calculated function begins to depart from $T_{\text {qad }}^{+}$around $3.5 \mathrm{~T}$. It is reasonable to admit that $T_{\text {ad }}^{+}(B)$ was not the best approximation to the true isentropic curve at high fields.

In consequence, $T_{\text {qad }}^{-}(B)$ from phase II (ordered magnetic phase) to phase IV (disordered magnetic phase) cannot be explained without finite $Q_{\text {neq }}^{-}(B)$ focused on a phase boundary as depicted in Fig. 5 and Fig. 6 . Since $\mathrm{Ce}_{1-x} \mathrm{La}_{x} \mathrm{~B}_{6}$ was the first group of materials which has been scrutinized in the framework of Section II, it might be premature to generalize the above arguments. However, we believe further investigations of the nonequilibrium MCE in various other systems with different experimental tools would deliver valuable information regarding magnetic phases and phase transitions. For instance, investigating magnetic relaxation or hysteresis loops would provide information regarding the relaxation of a non-equilibrium phase towards the equilibrium phase. Moreover, it is speculated from our analysis that there exists a region of phase transition in which order parameters are constantly competing, and scattering between constituent quasi-particles is easily enhanced by the time-varying field. Therefore, time-resolved microscopic inspection by using a scanning tunneling microscope, atomic force microscope, or magnetic force microscope etc., would be informative to obtain a deeper understanding related to non-equilibrium features of the MCE. Simultaneously, it will be worthwhile to verify whether the ansatz (Eq. (8)) is derivable from time-dependent many-body quantum theory or not. 


\section{CONCLUSION}

To summarize, the magnetocaloric effect (MCE) from $\mathrm{YbPt}_{2} \mathrm{Sn}$ shows a well defined paramagnetic hehavior below $4 \mathrm{~K}$. Because the material is metallic, we are free from a complicated procedure supplementing a poor thermal conductivity of previously used insulating paramagnets when building an adiabatic demagnetization refrigerator. The $\mathrm{MCE}$ in $\mathrm{YbPt}_{2} \mathrm{Sn}$ is reversible and well described by equilibrium thermodynamics. On the other hand, the MCE from $\mathrm{Ce}_{0.5} \mathrm{La}_{0.5} \mathrm{~B}_{6}$ is complicated. It is highly irreversible due to focused field-induced heating on the brink of a phase transition and antiferromagnetic domain motions. The practical model which includes analytic ansatzes of such non-equilibrium effects well described the observed field-hysteresis. We hope further investigation of various other magnetocaloric materials would unravel the correct mechanism behind the non-equilibrium MCE.

\section{APPENDIX}

Eddy current perturbation The correction factor corresponding to the eddy current heating was negligible for both cases discussed above. Starting from Maxwell's equation, $\nabla \times \vec{E}=-\frac{\partial \vec{B}}{\partial t}$, the eddy current power dissipation was derived as $P_{\text {eddy }}=\left(\frac{V R_{\mathrm{o}}^{2}}{8 \rho}\right) r^{2} \approx 10^{-12} \mathrm{~W}$, where $V=0.85 \mathrm{~cm}^{3}, R_{\circ}=3 \mathrm{~mm}$ (radius of the pil- lar), $\rho=250 \mu \Omega \mathrm{cm}$ (ref. Jang, Gruner). At $100 \mathrm{mK}$, $C_{p} \approx 1 \mathrm{~J} / \mathrm{mol} \cdot \mathrm{K}$ and heat capacity, $C$ of $10 \mathrm{~g}$ of $\mathrm{YbPt}_{2} \mathrm{Sn}$ pillar becomes about $0.0147 \mathrm{~J} / \mathrm{K}$. Given that it took $\Delta t=40 \mathrm{~min}$ for the field sweep down, estimated $T_{\text {eddy }} \approx P_{\text {eddy }} \times \Delta t / C=0.17 \mu \mathrm{K}$.

In the case of the $\mathrm{Ce}_{0.5} \mathrm{La}_{0.5} \mathrm{~B}_{6}$, cylindrical specimen with $R_{\circ}=1 \mathrm{~mm}, V=3.14 \times 10^{-4} \mathrm{~cm}^{3}$ was assumed. From the reported resistivity of $\mathrm{Ce}_{0.5} \mathrm{La}_{0.5} \mathrm{~B}_{6}$, $\rho=20 \mu \Omega \mathrm{cm}$ at $100 \mathrm{mK}$ was extrapolated. At the same temperature, the heat capacity, $C$, of $5 \mathrm{mg}$ of $\mathrm{Ce}_{0.5} \mathrm{La}_{0.5} \mathrm{~B}_{6}$ was $3.22 \mu \mathrm{J} / \mathrm{K}$. The roughly estimated increase of temperature for $40 \mathrm{~min}$ due to the eddy current heating is then, $T_{\text {eddy }} \approx 4 \mu \mathrm{K}$.

For both $\mathrm{YbPt}_{2} \mathrm{Sn}$ and $\mathrm{Ce}_{0.5} \mathrm{La}_{0.5} \mathrm{~B}_{6}, C_{p}$ and $\rho$ increase and a more accurate estimation of $T_{\text {eddy }}$ will be smaller. In fields, both $C_{p}$ and $\rho$ decrease and $T_{\text {eddy }}(B)$ will be similar to its zero field values. Therefore, given that we have dealt with a change in $T_{\text {qad }}(B)$ of the order of at least $1 \mathrm{mK}$, we can discard $T_{\text {eddy }}$ as evaluated above.

\section{ACKNOWLEDGEMENT}

D. J. appreciates useful discussions with Inseok Yang, Alistair S. Cameron, and Dmytro S. Inosov. D. J. acknowledges support from the project entitled Research on the redefinition of international system of units (SI) at Korea Institute of Standards and Science (KRISS). T. G. is funded by the Humboldt Foundation. Support was also provided by the German Research Foundation (DFG), Research Unit 960 Quantum Phase Transitions and Project No. BR 4110/1-1.
[1] P. Weiss and A. Piccard, J. Phys. (Paris) 5th Ser. 7, 103 (1917).

[2] A. Smith, Eur. J. Phys. H. 38, 507 (2013).

[3] P. Debye, Ann. Phys. (Berl.) 386, 1154 (1926).

[4] W. F. Giauque, J. Am. Chem. Soc. 49, 1864 (1927).

[5] W. F. Giauque and D. P. MacDougall, Phys. Rev. 43, 768 (1933).

[6] J. M. Daniels and N. Kurti, Proc. Royal Soc. London. Series. A, Math. Phys. Sci. 221, 243 (1954).

[7] E. W. Hornung, R. A. Fisher, G. E. Brodale, and W. F. Giauque, J. Chem. Phys. 61, 282 (1974).

[8] P. Timbie, G. Bernstein, and P. Richards, Cryogenics 30, 271 (1990).

[9] C. Hagmann, D. Benford, and P. Richards, Cryogenics 34, 213 (1994).

[10] P. Shirron, E. Canavan, M. DiPirro, J. Francis, M. Jackson, J. Tuttle, T. King, and M. Grabowski, Cryogenics 44, 581 (2004).

[11] J. Bartlett, G. Hardy, I. Hepburn, C. Brockley-Blatt, P. Coker, E. Crofts, B. Winter, S. Milward, R. StaffordAllen, M. Brownhill, J. Reed, M. Linder, and N. Rando, Cryogenics 50, 582 (2010).

[12] D. Jang, T. Gruner, A. Steppke, K. Mitsumoto, C. Geibel, and M. Brando, Nat. Commun. 6, 8680 (2015).

[13] P. Wikus, E. Canavan, S. T. Heine, K. Matsumoto, and T. Numazawa, Cryogenics 62, 150 (2014).

[14] X. Moya, S. Kar-Narayan, and N. D. Mathur, Nat. Mater. 13, 439 (2014).
[15] K. A. Gschneidner Jr., V. K. Pecharsky, and A. O. Tsokol, Rep. Prog. Phys. 68, 1479 (2005).

[16] A. Tomokiyo, H. Yayama, T. Hashimoto, T. Aomine, M. Nishida, and S. Sakaguchi, Cryogenics 25, 271 (1985).

[17] A. Smith, Adv. Energy Mater. 2, 1288 (2012).

[18] A. M. Tishin, K. A. Gschneidner, and V. K. Pecharsky, Phys. Rev. B 59, 503 (1999).

[19] A. M. Tishin and Y. I. Spichkin, The Magnetocaloric Effect and its Applications, Series in Condensed Matter Physics (IOP Publishing Ltd. Bristol and Philadelphia, 2003).

[20] Y. Tokiwa, T. Radu, C. Geibel, F. Steglich, and P. Gegenwart, Phys. Rev. Lett. 102, 066401 (2009).

[21] B. Wolf, Y. Tsui, D. Jaiswal-Nagar, U. Tutsch, A. Honecker, K. Remović-Langer, G. Hofmann, A. Prokofiev, W. Assmus, G. Donath, and M. Lang, Proc. Natl. Acad. Sci. 108, 6862 (2011).

[22] D. Jang, P. Y. Portnichenko, A. S. Cameron, G. Friemel, A. V. Dukhnenko, N. Y. Shitsevalova, V. B. Filipov, A. Schneidewind, A. Ivanov, D. S. Inosov, and M. Brando, npj Quantum Mater. 2, 62 (2017).

[23] R. K. Pathria, Statistical Mechanics 2nd ed. (Butterworth - Heinemann, An imprint of Elsevier Science, Linacre House, Jordan Hill, Oxford, 1996).

[24] R. J. Baxter, Exactly Solved Models in Statistical Mechanics (Academic Press Ltd. London and San Diego, 1989).

[25] K. Momma and F. Izumi, J. Appl. Cryst. 44, 1272 (2011).

[26] T. Gruner, D. Jang, A. Steppke, M. Brando, F. Ritter, C. Krellner, and C. Geibel, J. Phys.: Condens. Matter. 
26, 485002 (2014).

[27] G. Friemel, Y. Li, A. Dukhnenko, N. Shitsevalova, N. Sluchanko, A. Ivanov, V. Filipov, B. Keimer, and D. Inosov, Nat. Commun. 3, 830 (2012).

[28] N. N. Sirota, V. V. Novikov, V. A. Vinokurov, and Y. B. Paderno, Phys. Solid State 40, 1856 (1998).

[29] N. N. Sirota, V. V. Novikov, and A. V. Novikov, Phys. Solid State 42, 2093 (2000).

[30] T. Gürel and R. Eryiğit, Phys. Rev. B 82, 104302 (2010).

[31] R. Shiina, H. Shiba, and P. Thalmeier, J. Phys. Soc. Jpn. 66, 1741 (1997).

[32] R. Shiina, O. Sakai, H. Shiba, and P. Thalmeier, J. Phys. Soc. Jpn. 67, 941 (1998).

[33] A. S. Cameron, G. Friemel, and D. S. Inosov, Rep. Prog. Phys. 79, 066502 (2016).

[34] S. E. Nikitin, P. Y. Portnichenko, A. V. Dukhnenko, N. Y. Shitsevalova, V. B. Filipov, Y. Qiu, J. A. RodriguezRivera, J. Ollivier, and D. S. Inosov, Phys. Rev. B 97, 075116 (2018).

[35] G. Friemel, H. Jang, A. Schneidewind, A. Ivanov, A. V. Dukhnenko, N. Y. Shitsevalova, V. B. Filipov, B. Keimer, and D. S. Inosov, Phys. Rev. B 92, 014410 (2015).

[36] H. Wilhelm, T. Lühmann, T. Rus, and F. Steglich, Rev. Sci. Instr. 75, 2700 (2004).

[37] T. Inui, Y. Tanabe, and Y. Onodera, Group Theory and Its Application in Physics, Springer Series in SolidState Sciences 78 (Springer-Verlag, Berlin and Heidelberg, 1990).

[38] O. E. Vilches and J. C. Wheatley, Phys. Rev. 148, 509 (1966).

[39] P. Schiffer, A. P. Ramirez, D. A. Huse, and A. J. Valentino, Phys. Rev. Lett. 73, 2500 (1994).

[40] D. P. Goshorn, D. G. Onn, and J. P. Remeika, Phys. Rev. B 15, 3527 (1977).

[41] P. J. von Ranke, N. A. de Oliveira, M. V. T. Costa, E. Nobrega, A. Caldas, and I. G. de Oliveira, J. Magn. Magn. Mater. 226-230, 970 (2001).

[42] P. J. von Ranke, I. G. de Oliveira, A. P. G. aes, and X. A. da Silva, Phys. Rev. B 61, 447 (2000).

[43] T. A. Yamamoto, T. Nakagawa, K. Sako, T. Arakawa, and H. Nitani, J. Alloy. Compd. 376, 17 (2004).
[44] T. Nakagawa, K. Sako, T. Arakawa, and T. A. Yamamoto, J. Alloy. Compd. 364, 53 (2004).

[45] P. J. von Ranke and D. F. Grangeia, J. Appl. Phys. 93, 4055 (2003).

[46] P. J. von Ranke, E. P. Nóbrega, I. G. de Oliveira, A. M. Gomes, and R. S. Sarthour, Phys. Rev. B 63, 184406 (2000).

[47] A. Cho, Science 326, 778 (2009).

[48] J. Glanz, Science 279, 2045 (1998).

[49] E. Zirngiebl, B. Hillebrands, S. Blumenröder, G. Güntherodt, M. Loewenhaupt, J. M. Carpenter, K. Winzer, and Z. Fisk, Phys. Rev. B 30, 4052 (1984).

[50] O. Sakai, R. Shiina, H. Shiba, and P. Thalmeier, J. Phys. Soc. Jpn. 66, 3005 (1997).

[51] H. Nakao, K. Magishi, Y. Wakabayashi, Y. Murakami, K. Koyama, K. Hirota, Y. Endoh, , and S. Kunii, J. Phys. Soc. Jpn. 70, 1857 (2001).

[52] M. Sera, H. Ichikawa, T. Yokoo, J. Akimitsu, M. Nishi, K. Kakurai, and S. Kunii, Phys. Rev. Lett. 86, 1578 (2001).

[53] M. Takigawa, H. Yasuoka, T. Tanaka, and Y. Ishizawa, J. Phys. Soc. Jpn. 52, 728 (1983).

[54] J. M. Effantin, J. Rossat-Mignod, P. Burlet, H. Bartholin, S. Kunii, and T. Kasuya, J. Magn. Magn. Mater. 47 \& 48, 145 (1985).

[55] O. Zaharko, P. Fischer, A. Schenck, S. Kunii, P.-J. Brown, F. Tasset, and T. Hansen, Phys. Rev. B 68, 214401 (2003).

[56] M. Sera and S. Kobayashi, J. Phys. Soc. Jpn. 68, 1664 (1999).

[57] D. Mannix, Y. Tanaka, D. Carbone, N. Bernhoeft, and S. Kunii, Phys. Rev. Lett. 95, 117206 (2005).

[58] K. Kuwahara, K. Iwasa, M. Kohgi, N. Aso, M. Sera, and F. Iga, J. Phys. Soc. Jpn. 76, 093702 (2007).

[59] T. Matsumura, T. Yonemura, K. Kunimori, M. Sera, F. Iga, T. Nagao, and J. Igarashi, Phys. Rev. B 85, 174417 (2012).

[60] T. Matsumura, S. Michimura, T. Inami, T. Otsubo, H. Tanida, F. Iga, and M. Sera, Phys. Rev. B 89, 014422 (2014). 